\title{
RECONFIGURABLE CONTROL FOR A SCARA ROBOT USING RBF NETWORKS
}

\author{
Mohamed Salah Khireddine — Abdelhalim Boutarfa
}

\begin{abstract}
Faults in an industrial process could be timely detected and diagnosed in many cases. It is possible to subsequently reconfigure the control system so that it can safely continue its operation (possibly with degraded performance) until the time comes when it can be switched off for maintenance. In order to minimize the chances for drastic events such as a complete failure, safety-critical systems must possess the properties of increased reliability and safety. Faults in robotic systems are inevitable. They have diverse characteristics, magnitudes and origins, from the familiar viscous friction to Coulomb/Sticktion friction, and from structural vibrations. This paper presents an on-line environmental fault detection, isolation and an accommodation scheme.
\end{abstract}

K e y w or d s: fault diagnosis, robotics, scara robot arm, fault isolation, fault tolerant control

\section{INTRODUCTION}

The effectiveness of the proposed approach is verified by the development of the FTC scheme for a SCARA robot. Results of this extensive numerical study are included to verify the applicability of the proposed scheme. During the detection stage, faults are monitored and detected using a detection/approximation observer, which is robust with respect to unmodelled dynamics.

The detection/approximation observer is also used to approximate changes whose dynamics are not found to be equivalent to any a-priori known change scenarios. The dynamics of the fault can be approximated using on-line approximation techniques, which include: multilayer neural networks, polynomials, rational functions, spline functions, radial-basis-function (RBF) networks, adaptive fuzzy systems, [1] [2].

From the past experience, RBF networks performed very well in robotic applications. For this reason, they are employed in this paper for approximation purposes [2] [3]. Section 2 describes the dynamic model of the robotic system and of the faults. The general framework of the proposed scheme is studied in section 3. This section thoroughly investigates every stage of FTC. Simulation studies are presented in section 4.

Faults can be separated into two distinct categories: those that change the nonlinear dynamics of the nominal model, and those that do not. The second category depends only on time, and not on the states or the inputs, and therefore can be modelled as additive.

There are very effective techniques that can accommodate such faults, which include robust control and adaptive control [11-12]. Faults which belong to the first category have nonlinear dynamics and are have superior capabilities than conventional techniques. They are more difficult to handle because they depend both on the system's states and the input control signals. The purpose of our research work is to design a very effective method that specifically deals with the system state and input dependent faults, while being robust with respect to the unmodeled dynamics.

\section{DYNAMIC MODELS}

This section presents the well-studied dynamic structure of the robotic system. The second part of this section, concentrates on the dynamical structure, configuration and nomenclature of the changes (faults) in the robotic system. Innovations like parametric change history profiling and decoupled torque-dependent and state-dependent change model are introduced and thoroughly analyzed.

The dynamic motion of the robot arm in a robotic system is produced by the torques generated by the actuators. This relationship between the input torques and the time rates of change of the robot arm components configurations, represent the dynamic model of the robotic system.

The dynamic model of the robotic system can be derived using either Lagrangian, or Newton-Euler methods [3]. Both methods lead to the identical system of differential equations, which have been extensively studied in the literature on robots [3-6].

A general healthy n-degree of freedom robotic system is described by the following system of differential equations

$$
M(\theta) \ddot{\theta}+V(\theta, \dot{\theta})+G(\theta)+\mu(\theta, \dot{\theta}, \tau, t)=\tau
$$

where $\theta, \dot{\theta}, \ddot{\theta} \in R^{n}$ denote the vectors of joint positions, velocities, and accelerations, respectively, $\tau \epsilon \in R^{n}$ is the vector of input torques, $G(\theta) \in R^{n}$ is the vector of gravitational torque, $V(\theta, \dot{\theta}) \in R^{n}$ is the vector representing Coriolis and centripetal forces, $M(\theta) \in R^{n \times n}$ is the inertia matri x whose inverse exists, and $\mu(\theta, \dot{\theta}, \tau, t) \in$ $R^{n}$ denotes the unmodeled dynamics. It is assumed that the unmodeled dynamics are bounded.

\footnotetext{
* Advanced Electronics Laboratory (LEA), Chahid Boukhlouf Road, University of Batna, 05000, Algeria; mkhireddine@yahoo.fr, boutarfahal@yahoo.fr
} 


\subsection{Robotic system}

There are faults, which are referred to as drastic. They affect the system in such a way that it cannot function any further, and any ordinary control techniques cannot counteract their effects. An example of component catastrophic fault is a break of a joint or a link section. An example of actuator catastrophic fault is a short circuit in electric motor, permanently damaging the wiring. This type of faults is the worse case fault scenario and its effects on the system are obviously devastating. The only way they can be corrected is by direct operator (human) involvement and replacement of the system components. This paper concentrates only on the faults of smaller magnitudes, or non-drastic, which can be accommodated with ordinary control techniques. This type of faults includes different variations of friction, misbalances in the joint or actuator, the interaction with the external, etc. These faults can significantly affects the system's performance as well, which can be expressed in the loss of productivity, reduced life expectancy of the system, and unsafe environment for people and the external environment.

Thus, the presence of faults (non- catastrophic) is both state and time dependent, and their presence and magnitude is affected by a number of parameters. A general representation of the fault dynamics is taken to be

$$
F(\theta, \dot{\theta}, \tau, t)=\beta(P-p) f(\theta, \dot{\theta}, \tau)
$$

where, $f(\theta, \dot{\theta}, \tau) \in R^{n}$ denotes the fault dynamics, and $\beta(P-p) \in R^{n \times n}$ represents the state and/or time dependent fault profile that has the following structure

$$
\begin{gathered}
F(\theta, \dot{\theta}, \tau, t)=\operatorname{diag}\left[\beta_{1}\left(P_{1}-p_{1}\right), \beta_{2}\left(P_{2}-p_{2}\right),\right. \\
\left.\ldots, \beta_{n}\left(P_{n}-p_{n}\right)\right] \\
\beta_{j}\left(P_{j}-p_{j}\right)=\left\{\begin{array}{l}
1 \text { if } p_{j} \in P_{j} \\
0 \text { otherwise }
\end{array}\right.
\end{gathered}
$$

$\beta_{j}\left(P_{j}-p_{j}\right)$ represents the state and time history of the fault in the $j^{t} h$ state, $p_{j}$ is some parameter (for example time, or velocity), and $P_{j}$ is a region in this parameter history where the fault is present. The instance of the fault is declared when the value of the $p_{j}$ traverses into the $P_{j}$ region.

\subsubsection{Fault Dynamics}

Each fault is assumed to be linearly parameterized, which can be expressed in the following form

$$
f_{m}(\theta, \dot{\theta}, t)=\left[\begin{array}{c}
\sum_{i=1}^{s} c^{m}{ }_{1_{i}} w^{m}{ }_{1_{i}}\left(\theta_{1}, \dot{\theta}_{1}, \tau_{1}\right) \\
\sum_{i=1}^{s} c^{m}{ }_{2_{i}} w^{m}{ }_{2_{i}}\left(\theta_{2}, \dot{\theta}_{2}, \tau_{2}\right) \\
\cdot \\
\cdot \\
\sum_{i=1}^{s} c^{m}{ }_{n_{i}} w^{m}{ }_{n_{i}}\left(\theta_{n}, \dot{\theta}_{n}, \tau_{n}\right)
\end{array}\right]=
$$

$$
\begin{aligned}
& \sum_{i=1}^{s}\left[\begin{array}{c}
c^{m}{ }_{1_{i}} w^{m}{ }_{1_{i}}\left(\theta_{1}, \dot{\theta}_{1}, \tau_{1}\right) \\
c^{m}{ }_{2_{i}} w^{m}{ }_{2_{i}}\left(\theta_{2}, \dot{\theta}_{2}, \tau_{2}\right) \\
\cdot \\
\cdot \\
c^{m}{ }_{n_{i}} w^{m}{ }_{n_{i}}\left(\theta_{n}, \dot{\theta}_{n}, \tau_{n}\right)
\end{array}\right]= \\
& \sum_{i=1}^{s}\left[\begin{array}{ccccc}
c_{1 i}{ }^{m} & 0 & \cdot & \cdot & 0 \\
0 & c^{m}{ }_{2_{i}} & \cdot & \cdot & 0 \\
\cdot & \cdot & \cdot & \cdot & \cdot \\
\cdot & \cdot & \cdot & \cdot & \cdot \\
0 & 0 & \cdot & \cdot & c^{m}{ }_{n_{i}}
\end{array}\right]\left[\begin{array}{c}
w^{m}{ }_{1_{i}}\left(\theta_{1}, \dot{\theta}_{1}, \tau_{n}\right) \\
w^{m}{ }_{2_{i}}\left(\theta_{2}, \dot{\theta}_{2}, \tau_{2}\right) \\
\cdot \\
\cdot \\
w^{m}{ }_{n_{i}}\left(\theta_{n}, \dot{\theta}_{n}, \tau_{n}\right)
\end{array}\right]= \\
& =\sum_{i=1}^{s} \operatorname{diag}\left[C_{m_{i}}\right] W_{m_{i}}\left(\theta_{n}, \dot{\theta}_{n}, \tau_{n}\right)
\end{aligned}
$$

for $\quad m=1,2, \ldots, 2 N-1$

where $C_{m_{i}} \in R^{n}$ is a vector of the weights or parameters and $W_{m_{i}}: R^{n} \times R^{n} \times R^{+} R^{n}$ is a vector of dynamic functions.

\subsubsection{State and Torque-dependent Faults}

The fault dynamics can be represented as

$$
f_{m}(\theta, \dot{\theta}, \tau, t)=f_{m \theta}(\theta, \dot{\theta})+f_{m \tau}(\tau)
$$

where $f_{m \tau}(\tau)$ and $f_{m \theta}(\theta, \dot{\theta})$ represent torque-dependent and state-dependent faults respectively.

Summarizing this section's analysis of the faults in the robotic system, we arrive at the following comprehensive model of the robotic system

$$
\begin{gathered}
\underbrace{M(\theta) \ddot{\theta}+V(\theta, \dot{\theta})+G(\theta)+\mu(\theta, \dot{\theta}, \tau, t)}_{\text {Robotic System Dynamics }}+ \\
+\underbrace{\beta(P-p)\left[f_{m \theta}(\theta, \dot{\theta})+f_{m \tau}(\tau)\right]}_{\text {Fault Dynamics }}=\underbrace{\tau}_{\text {Input Torque }}
\end{gathered}
$$

\section{DETECTION/APPROXIMATION OBSERVERS}

The detection/approximation observer is a multifunction mechanism that bonds the entire FTC scheme together. While the system is healthy it is used to monitor it for faults and detect them if they do occur. During the subsequent stages, it is used to approximate and accommodate unknown fault dynamics, and to monitor the system for fault absence. Each of the detection/approximation observer application becomes evident in later sections. It is carefully designed to be robust with respect to unmodeled dynamics, and state and torquedependent faults.

The approximated torque-dependent and state-dependent fault dynamics in an $\mathrm{n}$ degree of freedom system can be represented by the following equations 


$$
\begin{aligned}
& \hat{f}_{\tau}(\tau, t)= \\
& =\left[\begin{array}{c}
h_{1}(t) \tau_{1} \\
h_{2}(t) \tau_{2} \\
\cdot \\
\cdot \\
h_{n}(t) \tau_{n}
\end{array}\right]\left[\begin{array}{ccccc}
h_{1}(t) & 0 & \cdot & \cdot & 0 \\
0 & h_{2}(t) & \cdot & \cdot & 0 \\
\cdot & \cdot & \cdot & \cdot & \cdot \\
\cdot & \cdot & \cdot & \cdot & \cdot \\
0 & 0 & \cdot & \cdot & \cdot \\
\cdot & \cdot & \cdot & \cdot & h_{n}(t)
\end{array}\right]\left[\begin{array}{c}
\tau_{1} \\
\tau_{2} \\
\cdot \\
\cdot \\
\tau_{n}
\end{array}\right]= \\
& =\operatorname{diag}[H(t)] \tau \\
& \hat{f}_{\theta}(\theta, \dot{\theta}, t)= \\
& =\left[\begin{array}{c}
\sum_{i=1}^{k} l_{1_{i}}(t) q_{1_{i}}\left(\theta_{1}\right) \\
\sum_{i=1}^{k} l_{2_{i}}(t) q_{2_{i}}\left(\theta_{2}\right) \\
\cdot \\
\cdot \\
\sum_{i=1}^{k} l_{n_{i}}(t) q_{n_{i}}\left(\theta_{n}\right)
\end{array}\right]+\left[\begin{array}{c}
\sum_{i=1}^{k} s_{1_{i}}(t) z_{1_{i}}\left(\dot{\theta}_{1}\right) \\
\sum_{i=1}^{k} s_{2_{i}}(t) z_{2_{i}}\left(\dot{\theta}_{2}\right) \\
\cdot \\
\cdot \\
\sum_{i=1}^{k} s_{n_{i}}(t) z_{n_{i}}\left(\dot{\theta}_{n}\right)
\end{array}\right]= \\
& =\sum_{i=1}^{k}\left[\begin{array}{c}
l_{1_{i}}(t) q_{1_{i}}\left(\theta_{1}\right) \\
l_{2_{i}}(t) q_{2_{i}}\left(\theta_{2}\right) \\
\cdot \\
\cdot \\
l_{n_{i}}(t) \dot{q}_{n_{i}}\left(\theta_{n}\right)
\end{array}\right]+\sum_{i=1}^{k}\left[\begin{array}{c}
s_{1_{i}}(t) z_{1_{i}}\left(\dot{\theta}_{1}\right) \\
s_{2_{i}}(t) z_{2_{i}}\left(\dot{\theta}_{2}\right) \\
\cdot \\
\cdot \\
s_{n_{i}}(t) z_{n_{i}}\left(\dot{\theta}_{n}\right)
\end{array}\right]= \\
& =\sum_{i=1}^{k}\left(\operatorname{diag}\left[L_{i}(t)\right] Q_{i}(\theta)+\operatorname{diag}\left[S_{i}(t)\right] Z_{i}(\theta)\right) \text {, }
\end{aligned}
$$

where $H(t) \in R^{n}, L_{t}(t) \in R^{n}$ and $S_{i}(t) \in R^{n}$ are the vectors of the weights or parameters. In equation (7) the velocity and the position dynamics are decoupled for analytical purposes. It does not affect the approximation effort, although it allows detecting the position-dependent faults and the velocity-dependent faults individually. Both velocity dependent and position-dependent dynamics of the fault are approximated using RBF neural network structures composing the $Q_{i}(\theta) \in R^{n}$ and $Z_{i}(\dot{\theta}) \in R^{n}$ vectors, and are structured as follows

$$
\begin{gathered}
q_{i j}\left(\theta_{j}\right)=\exp \left(-\frac{\left(\theta_{j}-a_{i j}\right)^{2}}{\sigma_{i j}{ }^{2}}\right), \\
z_{i j}\left(\theta_{j}\right)=\exp \left(-\frac{\left(\theta_{j}-b_{i j}\right)^{2}}{\omega_{i j}{ }^{2}}\right) \\
\text { for }=\left\{\begin{array}{l}
i=1,2, \ldots, k \\
j=1,2, \ldots, n
\end{array}\right.
\end{gathered}
$$

where $a_{i j}, b_{i j} \sigma_{i j}$ and $\omega_{i j}$ are the parameters of these networks [2], [7].

The detection/approximation observer is proposed

$$
\begin{aligned}
& \ddot{\widehat{\theta}}=-M^{-1}(V+G)+M^{-1}(I-\operatorname{diag}[H]) \tau \\
& -M^{-1} \sum_{i=1}^{k}\left(\operatorname{diag}\left[L_{i}\right] Q_{i}+\operatorname{diag}\left[S_{i}\right] Z_{i}\right)-\gamma(\dot{\hat{\theta}}-\dot{\theta})
\end{aligned}
$$

where $\gamma=\operatorname{diag}\left[\gamma_{1}, \gamma_{2}, \ldots, \gamma_{n}\right]$ is a positive definite stability matrix $[4-5]$.

\subsection{Detection}

Let $e_{o}=\grave{\theta}-\dot{\theta}$ denote the state estimation error, which will serve also as the residual vector [2], [5]. During the detection stage, the FTC monitors the system for the presence of the faults. While the system is healthy or no fault is present, the true system dynamics is represented as follows

$$
\dot{\theta}=-M^{-1}(V+G)+M^{-1} \tau
$$

While the system is healthy, the approximation model has the following form

$$
\ddot{\widetilde{\theta}}=-M^{-1}(V+G)+M^{-1} \tau-\gamma(\dot{\widehat{\theta}}-\dot{\theta})
$$

The dynamics of the estimation error in this case will be equal to

$$
\dot{e}=-\gamma e .
$$

The unmodeled dynamics $\eta$ are always present in the system, and can be mistakenly identified as a fault. In order to avoid such false alarms and to improve performance, a detection threshold is introduced. Prior to the fault occurrence, from the equation (9) the error equation is given by

$$
\begin{aligned}
& \dot{e}=-\gamma e+M^{-1} \eta \Longrightarrow e(t)=\exp (-\gamma) e_{0}(0)+ \\
& +\int_{0}^{t} \exp (-\gamma(t-T)) M^{-1}(T) \eta(T) \mathrm{d} T
\end{aligned}
$$

By introducing the upper bound on each element of $\eta \square$, given by $\left(\eta_{0}\right)_{j}=\sup \left|\eta_{j}\right| \square$, and taking into account $e(0)=0 \square$, we obtain the following equation

$$
\begin{gathered}
e(t) \leq \int_{0}^{t} \exp (-\gamma(t-T))\left(M^{-1}(T) \eta_{0}\right) \mathrm{d} T \\
D(\theta, \dot{\theta}, t)=\int_{0}^{t} \exp \left((-\gamma(t-T)) M^{-1}(T) \eta_{0}\right) \mathrm{d} T
\end{gathered}
$$

therefore, a fault is declared if

$$
\left|e_{j}\right|>d_{j}(t) ; \quad j=1,2, \ldots, n
$$

The value of $\eta_{0}$ may be found experimentally.

\subsection{Approximation}

$$
\begin{aligned}
\grave{H} & =H-H^{*}, \\
\grave{L}_{t} & =L_{t}-L^{*}, \\
\text { and } \quad \grave{S}_{t} & =S_{t}-S^{*} .
\end{aligned}
$$

Consequently we obtain

$$
\begin{array}{r}
\tilde{e}_{0}=-\gamma e_{0} M^{-1}[\operatorname{diag}[\check{H}] \tau+ \\
\left.\sum_{i=1}^{k}\left(\operatorname{diag}\left[\check{L}_{i}\right] Q_{i}+\operatorname{diag}\left[\check{S}_{i}\right] Z_{i}\right)-\eta\right]
\end{array}
$$

A Lyapunov function of the following form is employed 


$$
\begin{aligned}
U=\frac{1}{2} e_{0}^{T} e_{0} & +\frac{1}{2} \widetilde{H^{T}} T^{-1} \tilde{H}+\frac{1}{2} \sum_{i=1}^{k} \widetilde{L_{i}^{\Gamma}} \Psi^{-1} \widetilde{L_{i}} \\
& +\frac{1}{2} \sum_{i=1}^{k} \widetilde{S_{i}^{\Gamma}} Y^{-1} \widetilde{S_{i}} \geq 0
\end{aligned}
$$

where $\Gamma, \Psi, Y \in R^{R \times a}$ are adaptive gain matrices gains. Therefore

$$
\dot{U}=e_{0}^{T} \dot{e}_{0}+\dot{H^{T}} \Gamma^{-1} \tilde{H}+\sum_{i=1}^{k} \dot{L_{i}^{T}} \Psi^{-1} \widetilde{L_{i}}+\sum_{i=1}^{k} \dot{S}_{i}^{T} Y^{-1} \widetilde{S}_{i}
$$

By setting

$$
\begin{aligned}
& \dot{H}^{T} \Gamma^{-1}=e_{0}^{T} M^{-1} \operatorname{diag}[\tau] \text { or } \dot{H}=\Gamma \operatorname{diag}[\tau] M_{e_{0}}^{-1} \\
& \dot{L_{i}^{T}} \Psi^{-1}=e_{0}^{T} M^{-1} \operatorname{diag}\left[Q_{i}\right] \\
& \text { or } \\
& \dot{L}_{i}=\Psi \operatorname{diag}\left[Q_{i}\right] M_{e_{0}}^{-1}, \\
& \text { for } i=1,2, \ldots, k \\
& \dot{S_{i}^{T}} \Psi^{-1}=e_{0}^{T} M^{-1} \operatorname{diag}\left[Z_{i}\right] \text { or } \dot{S}_{i}=Y \operatorname{diag}\left[Z_{i}\right] M_{e_{0}}^{-1}
\end{aligned}
$$

we obtain

$$
\dot{U}=-e^{T} \gamma e-e^{T} M^{-1} \eta
$$

when $\eta=0$, we obtain: $\dot{U}=-e^{T} \gamma e \leq 0$ which is a negative semi-definite matrix, and therefore the approximation error will converge to zero. When $\eta \neq 0$, we obtain

$$
\dot{U}=-e_{0}^{T} \gamma e_{0}-e_{0}^{T} M^{-1} \eta .
$$

Following the previous analysis, the approximation observer equation will be

$$
\begin{array}{r}
\ddot{\hat{\theta}}=-M^{-1}(V+G)+M^{-1}(I+\operatorname{diag}[H]) \tau+ \\
M^{-1} \sum_{i=1}^{k}\left(\operatorname{diag}\left[L_{i}\right] Q_{i}+\operatorname{diag}\left[S_{i}\right] Z_{i}\right)-\gamma e
\end{array}
$$

\subsection{Accommodation}

Under healthy conditions, the nominal input torque $\tau=\tau_{0}$ is given by

$$
\begin{gathered}
\tau_{0}=M(\theta)\left\lfloor K_{P} \theta\left(\theta-\dot{\theta}_{d}\right)+K_{d}\left(\dot{\theta}-\dot{\theta}_{d}\right)+\ddot{\theta}_{d}\right\rfloor+ \\
V(\theta, \dot{\theta})+G(\theta)
\end{gathered}
$$

where $\theta_{d}, \dot{\theta}_{d}, \ddot{\theta}_{d} \in R^{n}$ are the vectors of desired joint positions, velocities, and accelerations, respectively, and $K_{p} \in R^{a \times n}$ and $K_{y} \in R^{n \times R}$ are negative definite matrices, which are designed, so that exponential convergence of the tracking errors is achieved. Applying the proposed torque and stage-dependent fault models, the input should have the following structure

$$
\tau=\left\{\begin{array}{l}
(I-\operatorname{diag}[H(t)])^{-1}\left(\tau_{0}+\hat{f}_{\theta}(\theta, \dot{\theta}, t)\right) \\
\tau_{0}
\end{array}\right.
$$

\section{SIMULATIONS RESULTS}

For simplicity, a three-link SCARA robot is utilized in this study. The dynamic equations, which can be derived via the Euler-Lagrangian method, are represented as follows

$$
\begin{aligned}
& {\left[\begin{array}{lll}
D_{11} & D_{12} & D_{13} \\
D_{21} & D_{22} & D_{23} \\
D_{31} & D_{32} & D_{33}
\end{array}\right]\left[\begin{array}{l}
\ddot{q}_{1} \\
\ddot{q}_{2} \\
\ddot{q}_{3}
\end{array}\right]} \\
& +l_{1} l_{2} \sin \left(q_{2}\right)\left[\begin{array}{lll}
C_{11} & C_{12} & C_{13} \\
C_{21} & C_{22} & C_{23} \\
C_{31} & C_{32} & C_{33}
\end{array}\right]\left[\begin{array}{l}
\dot{q}_{1} \\
\dot{q}_{2} \\
\dot{q}_{3}
\end{array}\right] \\
& +\left[\begin{array}{c}
0 \\
0 \\
-m_{3} g
\end{array}\right]
\end{aligned}
$$

where

$$
\begin{aligned}
& D_{11}=l^{2}{ }_{1}\left(\frac{m_{1}}{3}+m_{2}+m_{3}\right)+l_{1} l_{2}\left(m_{2}+\right. \\
& \left.+2 m_{3}\right) \cos \left(q_{2}\right)+l^{2}{ }_{2}\left(\frac{m_{2}}{3}+m_{3}\right) \\
& D_{13}=D_{23}=D_{31}=D_{33}=0 \\
& D_{12}=-l_{1} l_{2}\left(\frac{m_{2}}{2}+m_{3}\right) \cos \left(q_{2}\right)-\left(\frac{m_{2}}{3}+m_{3}\right)= \\
& =D_{21} \\
& D_{22}=l_{2}^{2}\left(\frac{m_{2}}{3}+m_{3}\right), \quad D_{33}=m_{3} \\
& C_{11}=-\dot{q}_{2}\left(m_{2}+2 m_{3}\right), \quad C_{12}=-\dot{q}_{2}\left(\frac{m_{2}}{2}+m_{3}\right) \\
& C_{13}=C_{22}=C_{23}=C_{31}=C_{32}=C_{33}=0
\end{aligned}
$$

in which $q_{1}, q_{2}$ and $q_{3}$ are the angles of joints 1,2 and 3; $m_{1}, m_{2}$ and $m_{3}$ are the mass of links 1,2 and $3 ; l_{1}, l_{2}$ and $l_{3}$ are the length of links 1,2 and $3 ; g$ is the gravity acceleration. Moreover, the system parameters of the SCARA robot are selected as:

$l_{1}=1.0 \mathrm{~m}, l_{2}=0.8 \mathrm{~m}, l_{3}=0.6 \mathrm{~m}, m_{1}=1.0 \mathrm{~kg}$, $m_{2}=0.8 \mathrm{~kg}, m_{3}=0.5 \mathrm{~kg}, g=9.8 \mathrm{kgms}^{-2}$.

This simulation study demonstrates that the presented scheme is effective when applied to a real life robotic system. The simulation was conducted using Matlab and Simulink.

In the joints (components), the most common and ever present type of faults is friction. Friction has been extensively analyzed and varieties of models are available. Friction models in the works by C. Canudas de Wit [8], [9], and [10] provide an excellent reflection of friction in the real joint

— Coulomb / Sticktion : $f(\dot{\theta})=\alpha \operatorname{sgn}(\dot{\theta})$

— Viscous : $f(\dot{\theta})=\alpha \dot{\theta}$ 


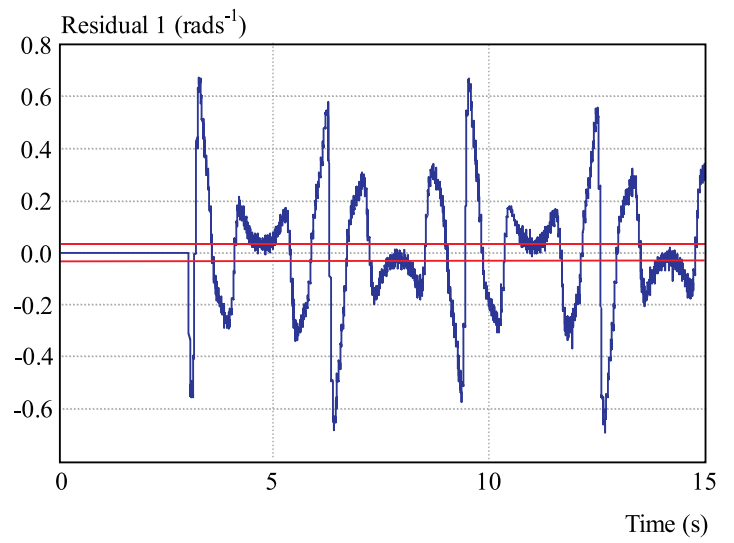

Fig. 1. Residual 1

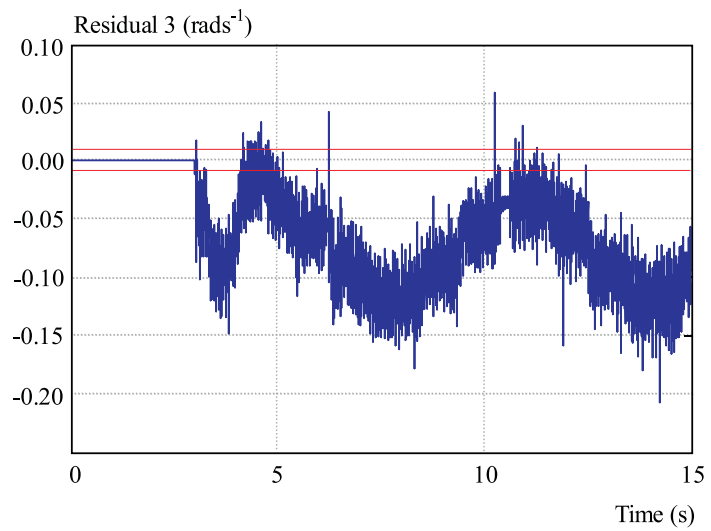

Fig. 3. Residual 3

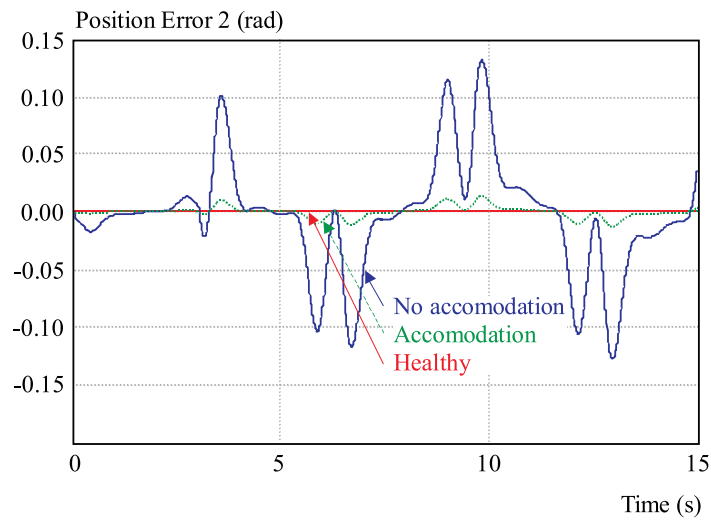

Fig. 5. Position Error 2

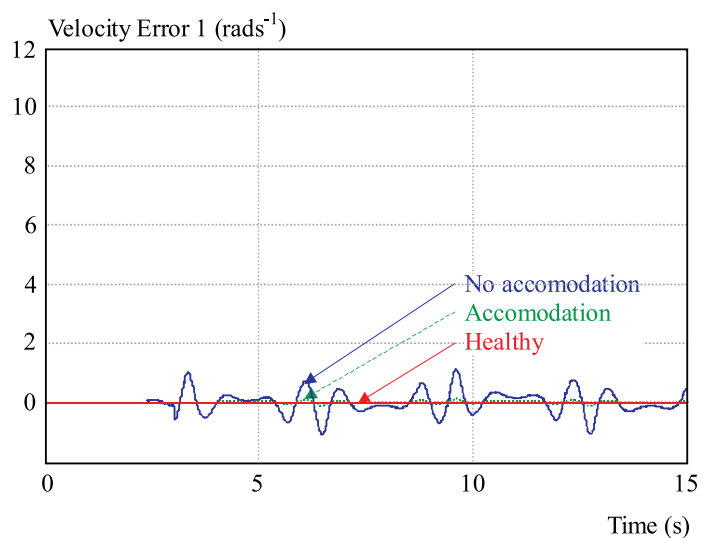

Fig. 7. Velocity Error 1

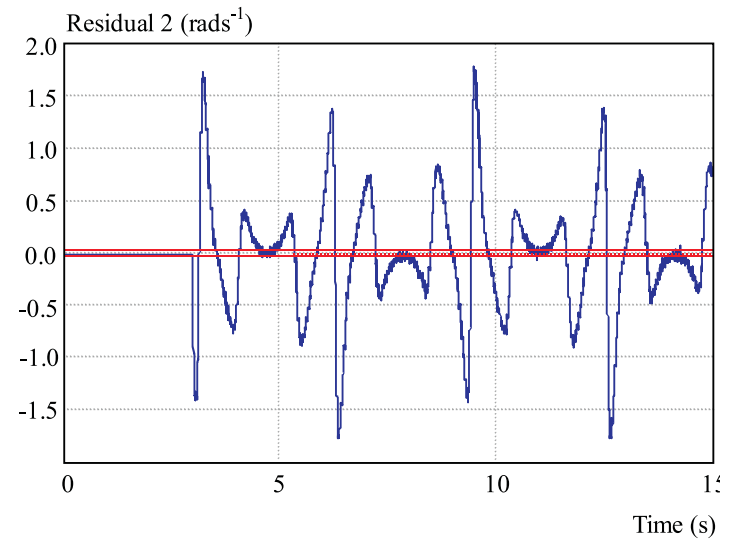

Fig. 2. Residual 2

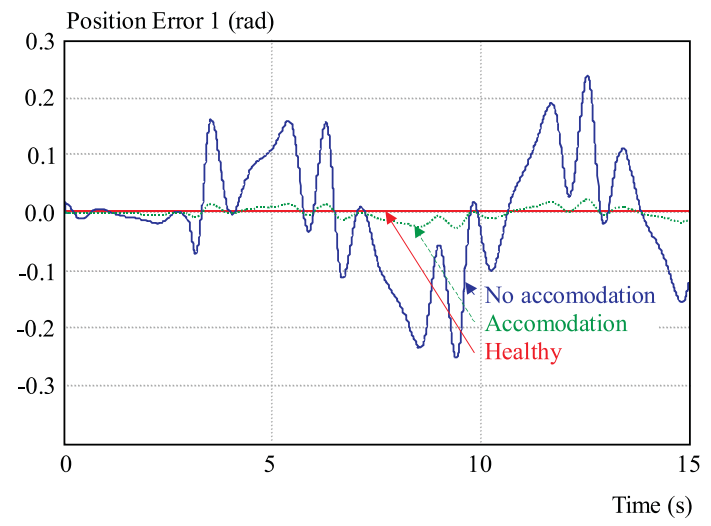

Fig. 4. Position Error 1

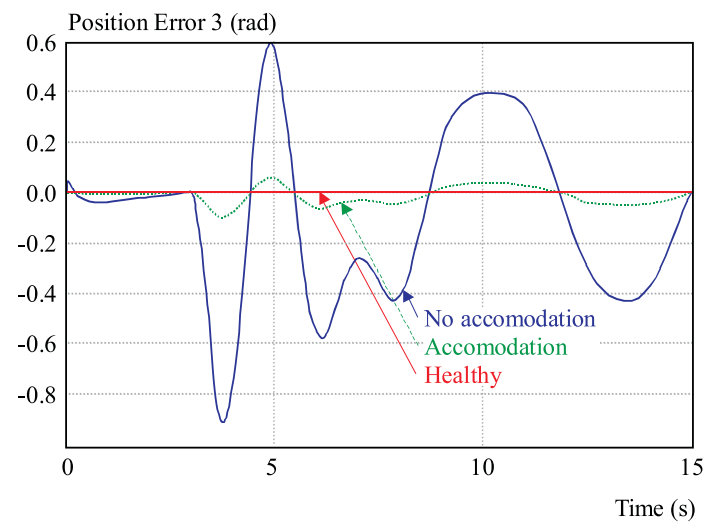

Fig. 6. Position Error 3

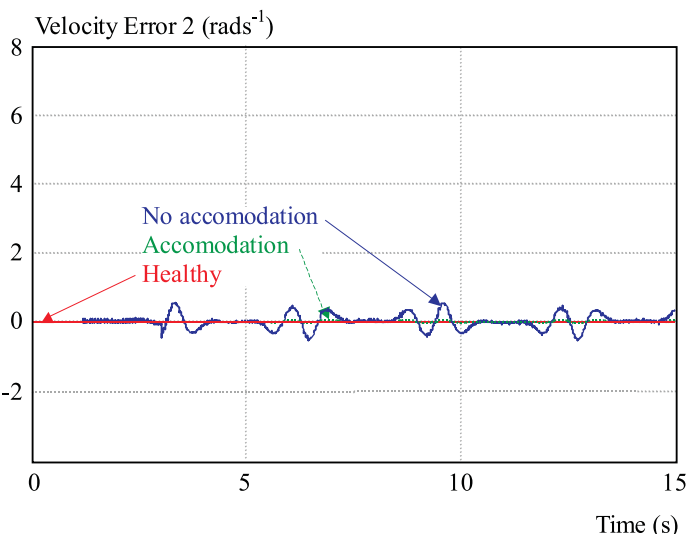

Fig. 8. Velocity Error 2 


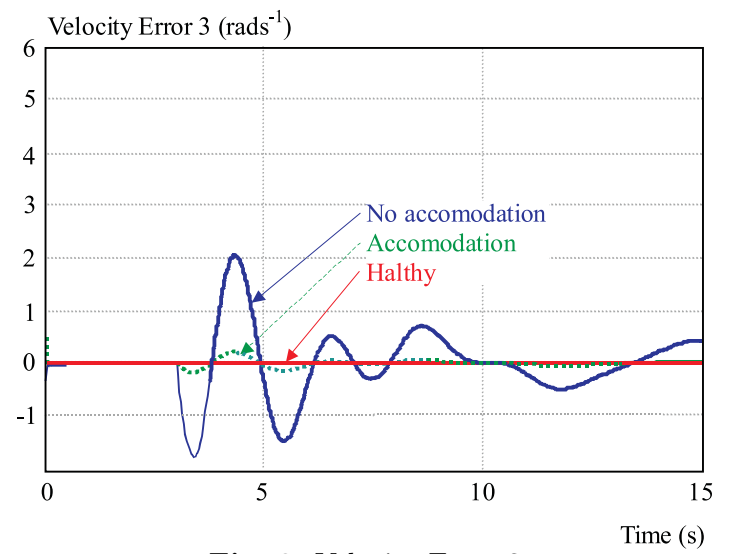

Fig. 9. Velocity Error 3

In SCARA manipulators, actuators are generally electric motors. Faults in rotating electric motors may be classified as electric faults, rotational faults and vibration faults

$$
\begin{array}{r}
f(\tau)=\alpha \tau, \\
-1<\alpha \leq K \leq \infty
\end{array}
$$

where $K$ is some maximum value that $\alpha$ can reach.

The results of simulations in the case of a healthy, but subject to uncertainties modeling show that residues vary and are moving away from zero. They are therefore sensitive to uncertainties in modeling. This creates false alarms and false detections.

We will now introduce the detection threshold defined by equation (15) to mount how it is possible to improve the detection of defects and reduce false alarms.

The first stage of the numerical study analyzes performance of the detection/approximation observer. Figure 1 to Fig. 9 demonstrate results of such study with an example of actuator and component fault detection and accommodation in a SCARA robot. The previously described fault dynamics are applied in this simulation.

\section{CONCLUSION AND FUTURE WORKS}

The new modeling technique was used to develop a very effective approach that both monitors the robotic system's health and its environment, and provides significant improvements to its performance. It is robust with respect to unmodeled dynamics. Detection, Isolation, and Accommodation (FTC) can be easily reshaped to work with a wide variety of systems and faults. One of the great advantages of the approach is that it can be applied to hydraulic, electrical or other types of robotic systems with minor modifications. This approach gives robotic system the tools to be aware of its constantly changing internal and external environment, identify or learn any faults, and accommodate them.

Maintenance is an important factor in the systems operation, especially in the areas where human access to the system is either limited or impossible. FTC transforms regular robotic system to a much more intelligent system, capable of self-monitoring and self-correcting. It provides the system with tools to eliminate or decrease the need for maintenance for non-catastrophic faults.

The above analysis has attempted to pull together theory from various management disciplines to help consider the issue of transfer of technology developed within a university setting. However, the framework is by no means particular to this area of technology, or to one university. It is therefore hoped that this framework can be used by academic entrepreneurs, whatever their discipline.

An important extension of this work is to implement a new algorithm to analyze the tolerance by using the local geometric properties obtained for each point in the operation process. Further work is needed within the proposed framework to improve provide flexible bandwidth adaptation and robustness.

\section{REFERENCES}

[1] KIM, Y. H.-LEWIS, F. L.: High-Level Feedback Control with Neural Network, World Scientific Publishing Co. Pte. Ltd.,(1998).

[2] POlyCARPoU, M. M.-HELMiCKI, A. J.: A. J., IEEE Transactions on Systems, Man, and Cybernetics 25 No. 11 (1995), 1447-1458.

[3] PATTON, R. J.: Fault tolerant control: The situation survey, IFAC SAFEPROCESS'97, Hull, UK, 2, (August 26-28, 1997), 1033-1055.

[4] ASSADA, H.-SLOtime, J. J. E. : Robot Analysis and Control, A Wiley Interscience, (1986).

[5] SLOtine, J. J. E.-LI, W. : Applied Nonlinear Control, Prentice Hall, (1991).

[6] SCHILlinG, J. R. : Fundamentals of Robotics. Analysis and Control, Prentice Hall, (1990).

[7] TERRA, M. H.-TINOS, R.: Fault Detection and Isolation in Robotic Manipulators via Neural Networks: A Comparison Among Three Architectures for Residual Analysis, Journal of Robotic Systems (2001), 357-374.

[8] CANUDAS de WIT, C.-OLSSON, H.-ASTROM, K. J.LISCHINSKY, P.: A New Model for Control of Systems with Friction, IEEE Transactions on Automatic Control 40 No. 3 (1995).

[9] CANUdAS de WIT, C.-GE, S. : Adaptive Friction Compensation for Systems with Generalized Velocity/Position Friction Dependency, IEEE Proceedings of the 36th Conference on Decision and Control, 1997, pp. 2465-2470.

10] BLANKE, M.-KINNAERT, M.-LUNZE, J.-STAROSWIECKI, M. : Diagnosis and Fault-Tolerant Control, Springer, 2-nd edition, 2006.

11] ZHANG, Y.-JIANG, J.: Issues on integration of fault diagnosis and reconfigurable control in active fault-tolerant control systems, Int. Proceedings of the IFAC Symposium SAFEPROCESS'06, Beijing, (2006).

12] POPESCU, M.-OLARU, I. B.-POPESCU, L.-GROFU, F. : The Simulation Hybrid Fuzzy Control of SCARA Robot, WSEAS Transactions on Systems and Control 3 No. 2 (February 2008).

Recieved 18 May 2009

Mohamed Salah Khireddine (Dr) was born in 1956 in Tolga, Algeria. He received the Computer Science Engineer 
Degree from the University of Algiers (1980) and his Doctorate (1990) in Automation and computer science from University of Marseille (France). He is currently Assistant Professor in Automation and Industrial Computing at Batna University, research member in LEA laboratory. He is also the head of Artificial Intelligence team in LRP laboratory. His research interests include Faults Diagnosis, Fault Tolerant Control, Artificial Intelligence, Control Systems, Pattern Recognition, Robotics and his applications.

Abdelhalim Boutarfa (Dr) was born in 1958 in Lyon, France. He is graduated from Constantine University in
Physics (Licence). He received the Electronics Engineer Degree from the Polytechnic School of Algiers (ENP), the Magister and Doctorate in Control from University of Batna. In 2007 he also obtained a postdoctoral degree in "Habilitation of conducting research in Control" from the same University. He is currently an Associate Professor, research member in LEA laboratory. His research interest includes applications of neural networks to pattern recognition, robotic systems, vision systems, and industrial processes. He focuses his research on intelligent pattern recognition, learning, analysis and intelligent control of large scale complex systems. 